Note on the Definition of Reflecting Telescopes and on the Images of Bright Stars on Photographic Plates. By Lieut.-General J. F. Tennant, R.E., F.R.S.

It has frequently been said that the images of stars are seen in reflecting telescopes without any diffraction rings surrounding them. This statement has always surprised me, because it is accompanied by the statement that the discs are very small compared with those in reflectors. The theoretical investigation which accounts for the rings assumes that the optical rays are rigorously convergent, and this is more true for a well-figured speculum than for an object-glass.

Recently, in looking over a translation of Foucault's mémoire on "The Construction of Silvered Glass Specula," I was struck by his statement that he always saw "traces of the rings," and I wrote to M. Henry, who, I believe, follows Foucault's methods almost entirely, inquiring what his experience was. M. Henry kindly informs me that not only does he always see rings with his reflectors, but that they are, in fact, easier to see than with a refractor. In his article on Light I find that Sir J. Herschel states that the rings surrounding star images were discovered by Sir W. Herschel, but he says that they are less easily seen than with a refractor.

I have since calculated the places and brilliancy of the rings to be seen in a telescope with an obstructing disc one-third of the diameter of the aperture, centrally placed, and the result has somewhat surprised me.

Calling $e$ the aperture of the telescope in English inches and $s$ the angular diameter of the ring in seconds, Sir George Airy gives for an open aperture the following values, the unit of brilliancy being the centre of the images :-

$\begin{array}{llccr}\text { es } & 2 \cdot 76 & 3 \cdot 70 & 5 \cdot 16 & 6 \cdot 09 \\ \text { Brilliancy } & 0 & \frac{1}{57} & 0 & \frac{1}{240}\end{array}$

With one-third stopped centrally as above, which would nearly represent a reflector giving a field of $2^{\circ}$ diameter, I find-

$\begin{array}{llclc}\text { es } & 2 \cdot 47 & 3.54 & 5.44 & 6 \cdot 22 \\ \text { Brilliancy } & 0 & \frac{1}{18} & 0 & \frac{1}{712}\end{array}$

It would follow that while the central image is smaller and also the first dark ring, the first bright ring would be far more conspicuous than in a refractor, both by its width and brilliancy, compared with the central image.

The place of the third dark ring would be very little inside the corresponding place in a refractor, and so would that of the 
third bright ring, which, too, would be less brilliant than in a refractor.

It would require a good deal of labour in interpolating to get the diameters and brilliancy of the rings in a reflector where the small speculum is, as usual, only large enough to show a central object, or about one-ninth of that of the speculum, but it is evident that these would be intermediate between the two sets of values above given, and that there can be no theoretical reason why rings should be absent if carefully looked for under suitable circumstances, provided the figure is good.

I would add that I believe that the great diameter of the images of large stars on photographs will be explained, at all events in part, by the fact that the visible image is by no means all the acting light. If a plate be exposed so as to show stars of the I $4^{\text {th }}$ magnitude on the Paris scale, then we shall have sensible impressions made by light whose intensity is $\frac{I}{2^{\cdot} 5^{13}}$ of that of a Ist magnitude star. Roughly, we may call this $\frac{1}{149000}$ of the light, the distance from the centre of the image at which this fails is far beyond the powers of the integral table given in Sir G. Airy's little book, and I doubt if it could be determined with any accuracy, for at so great a distance the spectra would overlap, and it would be necessary to estimate the total effect of range of those colours which are impressed on the plate. Manifestly, however, the photographic image would be very large, though it does not seem possible it can fill the great space we sometimes see.

Of course light diffused within the photographic film would add its effects to those of incident lights.

P.S.-In a more recent communication M. Henry tells me that they some time ago tried specula up to $50 \mathrm{~cm}$. (nearly 20 inches) with various sized patches, and under favourable circumstances found just such a brilliant ring as I have described.

\section{Note on Testing Polished Flat Surfaces.}

By A. A. Common, F.R.S.

The following method of testing flat surfaces has some advantages over the methods generally employed, and is particularly useful in enabling the errors to be localised exactly. It is an extension of Foucault's method of testing concave surfaces, and consists in putting on the flat surface to be tested, in the cone of rays coming from a concave surface in the process of testing by Foucault's method, and thus finding the effect of the reflection on the course of the rays.

The concave mirror should strictly be quite spherical and, of course, somewhat larger than the plane surface to be tested, but an ordinary speculum will do almost as well; any errors that 